\title{
More examples of pseudosymmetric braided categories *
}

\author{
Florin Panaite \\ Institute of Mathematics of the Romanian Academy \\ PO-Box 1-764, RO-014700 Bucharest, Romania \\ e-mail: Florin.Panaite@imar.ro \\ Mihai D. Staic ${ }^{\dagger}$ \\ Department of Mathematics and Statistics, BGSU \\ Bowling Green, OH 43403, USA \\ e-mail: mstaic@bgsu.edu
}

\begin{abstract}
We study some examples of braided categories and quasitriangular Hopf algebras and decide which of them is pseudosymmetric, respectively pseudotriangular. We show also that there exists a universal pseudosymmetric braided category.
\end{abstract}

\section{Introduction}

Braided categories have been introduced by Joyal and Street in [4 as natural generalizations of symmetric categories. Roughly speaking, a braided category is a category that has a tensor product with a nice commutation rule. More precisely, for every two objects $U$ and $V$ we have an isomorphism $c_{U, V}: U \otimes V \rightarrow V \otimes U$ that satisfies certain conditions. These conditions are chosen in such a way that for every object $V$ in the category there exists a natural way to construct a representation for the braid group $B_{n}$ on $V^{\otimes n}$, therefore the name braided categories. If we impose the extra condition $c_{V, U} c_{U, V}=i d_{U \otimes V}$ for all objects $U, V$ in the category, we recover the definition of symmetric categories. It is well known that symmetric categories can be used to construct representations for the symmetric group $\Sigma_{n}$.

Pseudosymmetric categories are a special class of braided categories and have been introduced in [9]. The motivation was the study of certain categorical structures called twines, strong twines and pure-braided structures (introduced in [1], [8] and [13]). A braiding on a strict monoidal category is called pseudosymmetric if it satisfies a sort of modified braid relation; any symmetric braiding is pseudosymmetric. One of the most intriguing results obtained in [9] was that the category of Yetter-Drinfeld modules over a Hopf algebra $H$ is pseudosymmetric if and only if $H$ is commutative and cocommutative. We proved in [10] that pseudosymmetric categories can be used to construct representations for the group $P S_{n}=\frac{B_{n}}{\left.P_{n}, P_{n}\right]}$, the quotient of the braid group by the commutator subgroup of the pure braid group. There exists also a Hopf algebraic analogue of pseudosymmetric braidings: a quasitriangular structure on a Hopf algebra is called pseudotriangular if it satisfies a sort of modified quantum Yang-Baxter equation.

\footnotetext{
${ }^{*}$ Research partially supported by the CNCSIS project "Hopf algebras, cyclic homology and monoidal categories", contract nr. 560/2009, CNCSIS code ID_69.

${ }^{\dagger}$ Institute of Mathematics of the Romanian Academy, PO-Box 1-764, RO-014700 Bucharest, Romania.
} 
In this paper we tie some lose ends from [9] and [10]. We study more examples of braided categories and quasitriangular Hopf algebras and decide when they are pseudosymmetric, respectively pseudotriangular. Namely, we prove that the canonical braiding of the category $\mathcal{L} \mathcal{R}(H)$ of Yetter-Drinfeld-Long bimodules over a Hopf algebra $H$ (introduced in [11]) is pseudosymmetric if and only if $H$ is commutative and cocommutative. We show that any quasitriangular structure on the $4 \nu$-dimensional Radford's Hopf algebra $H_{\nu}$ (introduced in [12]) is pseudotriangular. We analyze the positive quasitriangular structures $R(\xi, \eta)$ on a Hopf algebra with positive bases $H\left(G ; G_{+}, G_{-}\right)$(as defined in [6], [7]), where $\xi, \eta$ are group homomorphisms from $G_{+}$to $G_{-}$, and we present a list of necessary and sufficient conditions for $R(\xi, \eta)$ to be pseudotriangular. If $R(\xi, \eta)$ is normal (i.e. if $\xi$ is trivial) these conditions reduce to the single relation $\eta(u v)=\eta(v u)$ for all $u, v \in G_{+}$.

In the last section we recall the pseudosymmetric braided category $\mathcal{P S}$ introduced in 10 and we show that it is a universal pseudosymmetric category. More precisely, we prove that it satisfies two universality properties similar to the ones satisfied by the universal braid category $\mathcal{B}($ see $[5])$.

\section{Preliminaries}

We work over a base field $k$. All algebras, linear spaces, etc, will be over $k$; unadorned $\otimes$ means $\otimes_{k}$. For a Hopf algebra $H$ with comultiplication $\Delta$ we denote $\Delta(h)=h_{1} \otimes h_{2}$, for $h \in H$. For terminology concerning Hopf algebras and monoidal categories we refer to [5].

Definition 1.1 ([9]) Let $\mathcal{C}$ be a strict monoidal category and $c$ a braiding on $\mathcal{C}$. We say that $c$ is pseudosymmetric if the following condition holds, for all $X, Y, Z \in \mathcal{C}$ :

$$
\left(c_{Y, Z} \otimes i d_{X}\right)\left(i d_{Y} \otimes c_{Z, X}^{-1}\right)\left(c_{X, Y} \otimes i d_{Z}\right)=\left(i d_{Z} \otimes c_{X, Y}\right)\left(c_{Z, X}^{-1} \otimes i d_{Y}\right)\left(i d_{X} \otimes c_{Y, Z}\right) .
$$

In this case we say that $\mathcal{C}$ is a pseudosymmetric braided category.

Proposition 1.2 ([9]) Let $\mathcal{C}$ be a strict monoidal category and $c$ a braiding on $\mathcal{C}$. Then $c$ is pseudosymmetric if and only if the family $T_{X, Y}:=c_{Y, X} c_{X, Y}: X \otimes Y \rightarrow X \otimes Y$ satisfies the condition $\left(T_{X, Y} \otimes i d_{Z}\right)\left(i d_{X} \otimes T_{Y, Z}\right)=\left(i d_{X} \otimes T_{Y, Z}\right)\left(T_{X, Y} \otimes i d_{Z}\right)$ for all $X, Y, Z \in \mathcal{C}$.

Definition 1.3 ([9]) Let $H$ be a Hopf algebra and $R \in H \otimes H$ a quasitriangular structure. Then $R$ is called pseudotriangular if $R_{12} R_{31}^{-1} R_{23}=R_{23} R_{31}^{-1} R_{12}$.

Proposition 1.4 ([9]) Let $H$ be a Hopf algebra and let $R$ be a quasitriangular structure on $H$. Then $R$ is pseudotriangular if and only if the element $F=R_{21} R \in H \otimes H$ satisfies the relation $F_{12} F_{23}=F_{23} F_{12}$.

\section{Yetter-Drinfeld-Long bimodules}

For a braided monoidal category $\mathcal{C}$ with braiding $c$, let $\mathcal{C}^{\text {in }}$ be equal to $\mathcal{C}$ as a monoidal category, with the mirror-reversed braiding $\tilde{c}_{M, N}:=c_{N, M}^{-1}$, for all objects $M, N \in \mathcal{C}$. Directly from the definition of a pseudosymmetric braiding, we immediately obtain:

Proposition 2.1 Let $\mathcal{C}$ be a strict braided monoidal category. Then $\mathcal{C}$ is pseudosymmetric if and only if $\mathcal{C}^{\text {in }}$ is pseudosymmetric. 
Let $H$ be a Hopf algebra with bijective antipode $S$. Consider the category ${ }_{H} \mathcal{Y} \mathcal{D}^{H}$ of leftright Yetter-Drinfeld modules over $H$, whose objects are vector spaces $M$ that are left $H$ modules (denote the action by $h \otimes m \mapsto h \cdot m$ ) and right $H$-comodules (denote the coaction by $\left.m \mapsto m_{(0)} \otimes m_{(1)} \in M \otimes H\right)$ satisfying the compatibility condition

$$
(h \cdot m)_{(0)} \otimes(h \cdot m)_{(1)}=h_{2} \cdot m_{(0)} \otimes h_{3} m_{(1)} S^{-1}\left(h_{1}\right), \quad \forall h \in H, m \in M .
$$

It is a monoidal category, with tensor product given by

$$
h \cdot(m \otimes n)=h_{1} \cdot m \otimes h_{2} \cdot n, \quad(m \otimes n)_{(0)} \otimes(m \otimes n)_{(1)}=m_{(0)} \otimes n_{(0)} \otimes n_{(1)} m_{(1)} .
$$

Moreover, it has a (canonical) braiding given by

$$
\begin{aligned}
& c_{M, N}: M \otimes N \rightarrow N \otimes M, \quad c_{M, N}(m \otimes n)=n_{(0)} \otimes n_{(1)} \cdot m, \\
& c_{M, N}^{-1}: N \otimes M \rightarrow M \otimes N, \quad c_{M, N}^{-1}(n \otimes m)=S\left(n_{(1)}\right) \cdot m \otimes n_{(0)} .
\end{aligned}
$$

Consider also the category ${ }_{H}^{H} \mathcal{Y} \mathcal{D}$ of left-left Yetter-Drinfeld modules over $H$, whose objects are vector spaces $M$ that are left $H$-modules (denote the action by $h \otimes m \mapsto h \cdot m$ ) and left $H$-comodules (denote the coaction by $m \mapsto m^{(-1)} \otimes m^{(0)} \in H \otimes M$ ) with compatibility condition

$$
\left(h_{1} \cdot m\right)^{(-1)} h_{2} \otimes\left(h_{1} \cdot m\right)^{(0)}=h_{1} m^{(-1)} \otimes h_{2} \cdot m^{(0)}, \quad \forall h \in H, m \in M .
$$

It is a monoidal category, with tensor product given by

$$
h \cdot(m \otimes n)=h_{1} \cdot m \otimes h_{2} \cdot n, \quad(m \otimes n)^{(-1)} \otimes(m \otimes n)^{(0)}=m^{(-1)} n^{(-1)} \otimes m^{(0)} \otimes n^{(0)} .
$$

Moreover, it has a (canonical) braiding given by

$$
\begin{aligned}
& c_{M, N}: M \otimes N \rightarrow N \otimes M, \quad c_{M, N}(m \otimes n)=m^{(-1)} \cdot n \otimes m^{(0)}, \\
& c_{M, N}^{-1}: N \otimes M \rightarrow M \otimes N, \quad c_{M, N}^{-1}(n \otimes m)=m^{(0)} \otimes S^{-1}\left(m^{(-1)}\right) \cdot n .
\end{aligned}
$$

Proposition 2.2 ([2]) For the categories ${ }_{H} \mathcal{Y D}^{H}$ and ${ }_{H}^{H} \mathcal{Y D}$ with braidings as above, we have an isomorphism of braided monoidal categories $\left({ }_{H} \mathcal{Y} \mathcal{D}^{H}\right)^{i n} \simeq{ }_{H}^{H} \mathcal{Y D}$.

Proposition 2.3 ([9]) The canonical braiding of ${ }_{H} \mathcal{Y D}^{H}$ is pseudosymmetric if and only if $H$ is commutative and cocommutative.

As a consequence of Propositions 2.1, 2.2 and 2.3, we obtain:

Proposition 2.4 The canonical braiding of ${ }_{H}^{H} \mathcal{Y D}$ is pseudosymmetric if and only if $H$ is commutative and cocommutative.

We recall now the braided monoidal category $\mathcal{L} \mathcal{R}(H)$ defined in $[11$. The objects of $\mathcal{L} \mathcal{R}(H)$ are vector spaces $M$ endowed with $H$-bimodule and $H$-bicomodule structures (denoted by $h \otimes$ $m \mapsto h \cdot m, m \otimes h \mapsto m \cdot h, m \mapsto m^{(-1)} \otimes m^{(0)}, m \mapsto m^{<0>} \otimes m^{<1>}$, for all $\left.h \in H, m \in M\right)$, such that $M$ is a left-left Yetter-Drinfeld module, a left-right Long module, a right-right YetterDrinfeld module and a right-left Long module, i.e. (for all $h \in H, m \in M$ ):

$$
\left(h_{1} \cdot m\right)^{(-1)} h_{2} \otimes\left(h_{1} \cdot m\right)^{(0)}=h_{1} m^{(-1)} \otimes h_{2} \cdot m^{(0)},
$$




$$
\begin{aligned}
& (h \cdot m)^{<0>} \otimes(h \cdot m)^{<1>}=h \cdot m^{<0>} \otimes m^{<1>}, \\
& \left(m \cdot h_{2}\right)^{<0>} \otimes h_{1}\left(m \cdot h_{2}\right)^{<1>}=m^{<0>} \cdot h_{1} \otimes m^{<1>} h_{2}, \\
& (m \cdot h)^{(-1)} \otimes(m \cdot h)^{(0)}=m^{(-1)} \otimes m^{(0)} \cdot h .
\end{aligned}
$$

Morphisms in $\mathcal{L} \mathcal{R}(H)$ are $H$-bilinear $H$-bicolinear maps. $\mathcal{L} \mathcal{R}(H)$ is a strict monoidal category, with unit $k$ endowed with usual $H$-bimodule and $H$-bicomodule structures, and tensor product given by: if $M, N \in \mathcal{L} \mathcal{R}(H)$ then $M \otimes N \in \mathcal{L} \mathcal{R}(H)$ as follows (for all $m \in M, n \in N, h \in H$ ):

$$
\begin{aligned}
& h \cdot(m \otimes n)=h_{1} \cdot m \otimes h_{2} \cdot n, \quad(m \otimes n) \cdot h=m \cdot h_{1} \otimes n \cdot h_{2}, \\
& (m \otimes n)^{(-1)} \otimes(m \otimes n)^{(0)}=m^{(-1)} n^{(-1)} \otimes\left(m^{(0)} \otimes n^{(0)}\right), \\
& (m \otimes n)^{<0>} \otimes(m \otimes n)^{<1>}=\left(m^{<0>} \otimes n^{<0>}\right) \otimes m^{<1>} n^{<1>} .
\end{aligned}
$$

Moreover, $\mathcal{L R}(H)$ has a (canonical) braiding defined, for $M, N \in \mathcal{L R}(H), m \in M, n \in N$, by

$$
\begin{array}{ll}
c_{M, N}: M \otimes N \rightarrow N \otimes M, & c_{M, N}(m \otimes n)=m^{(-1)} \cdot n^{<0>} \otimes m^{(0)} \cdot n^{<1>}, \\
c_{M, N}^{-1}: N \otimes M \rightarrow M \otimes N, & c_{M, N}^{-1}(n \otimes m)=m^{(0)} \cdot S^{-1}\left(n^{<1>}\right) \otimes S^{-1}\left(m^{(-1)}\right) \cdot n^{<0>} .
\end{array}
$$

Proposition 2.5 The canonical braiding of $\mathcal{L} \mathcal{R}(H)$ is pseudosymmetric if and only if $H$ is commutative and cocommutative.

Proof. Assume that the canonical braiding of $\mathcal{L} \mathcal{R}(H)$ is pseudosymmetric. As noted in [1], ${ }_{H}^{H} \mathcal{Y D}$ with its canonical braiding is a braided subcategory of $\mathcal{L} \mathcal{R}(H)$, so the canonical braiding of ${ }_{H}^{H} \mathcal{Y} \mathcal{D}$ is pseudosymmetric; by Proposition 2.4 it follows that $H$ is commutative and cocommutative. Conversely, assume that $H$ is commutative and cocommutative. Then one can see that the two Yetter-Drinfeld conditions appearing in the definition of $\mathcal{L R}(H)$ become Long conditions, that is (2.1) and (2.3) become respectively

$$
\begin{aligned}
& (h \cdot m)^{(-1)} \otimes(h \cdot m)^{(0)}=m^{(-1)} \otimes h \cdot m^{(0)}, \\
& (m \cdot h)^{<0>} \otimes(m \cdot h)^{<1>}=m^{<0>} \cdot h \otimes m^{<1>} .
\end{aligned}
$$

Let now $X, Y, Z \in \mathcal{L} \mathcal{R}(H)$; we compute, for $x \in X, y \in Y, z \in Z$ :

$$
\begin{aligned}
& \left(c_{Y, Z} \otimes i d_{X}\right)\left(i d_{Y} \otimes c_{Z, X}^{-1}\right)\left(c_{X, Y} \otimes i d_{Z}\right)(x \otimes y \otimes z) \\
& =\left(c_{Y, Z} \otimes i d_{X}\right)\left(i d_{Y} \otimes c_{Z, X}^{-1}\right)\left(x^{(-1)} \cdot y^{<0>} \otimes x^{(0)} \cdot y^{<1>} \otimes z\right) \\
& =\left(c_{Y, Z} \otimes i d_{X}\right)\left(x^{(-1)} \cdot y^{<0>} \otimes z^{(0)} \cdot S^{-1}\left(\left(x^{(0)} \cdot y^{<1>}\right)^{<1>}\right)\right. \\
& \left.\otimes S^{-1}\left(z^{(-1)}\right) \cdot\left(x^{(0)} \cdot y^{<1>}\right)^{<0>}\right) \\
& \stackrel{\text { [2.6) }}{=}\left(c_{Y, Z} \otimes i d_{X}\right)\left(x^{(-1)} \cdot y^{<0>} \otimes z^{(0)} \cdot S^{-1}\left(x^{(0)<1>}\right) \otimes S^{-1}\left(z^{(-1)}\right) \cdot x^{(0)<0>} \cdot y^{<1>}\right) \\
& =\left(x^{(-1)} \cdot y^{<0>}\right)^{(-1)} \cdot\left[z^{(0)} \cdot S^{-1}\left(x^{(0)<1>}\right)\right]^{<0>} \\
& \otimes\left(x^{(-1)} \cdot y^{<0>}\right)^{(0)} \cdot\left[z^{(0)} \cdot S^{-1}\left(x^{(0)<1>}\right)\right]^{<1>} \\
& \otimes S^{-1}\left(z^{(-1)}\right) \cdot x^{(0)<0>} \cdot y^{<1>} \\
& \stackrel{2.5[2.6}{=} y^{<0>(-1)} \cdot z^{(0)<0>} \cdot S^{-1}\left(x^{(0)<1>}\right) \otimes x^{(-1)} \cdot y^{<0>(0)} \cdot z^{(0)<1>} \\
& \otimes S^{-1}\left(z^{(-1)}\right) \cdot x^{(0)<0>} \cdot y^{<1>}
\end{aligned}
$$




$$
\begin{aligned}
&\left(i d_{Z} \otimes c_{X, Y}\right)\left(c_{Z, X}^{-1} \otimes i d_{Y}\right)\left(i d_{X} \otimes c_{Y, Z}\right)(x \otimes y \otimes z) \\
&=\quad\left(i d_{Z} \otimes c_{X, Y}\right)\left(c_{Z, X}^{-1} \otimes i d_{Y}\right)\left(x \otimes y^{(-1)} \cdot z^{<0>} \otimes y^{(0)} \cdot z^{<1>}\right) \\
&=\left(i d_{Z} \otimes c_{X, Y}\right)\left(\left[y^{(-1)} \cdot z^{<0>}\right]^{(0)} \cdot S^{-1}\left(x^{<1>}\right) \otimes S^{-1}\left(\left[y^{(-1)} \cdot z^{<0>}\right]^{(-1)}\right) \cdot x^{<0>}\right. \\
&\left.\otimes y^{(0)} \cdot z^{<1>}\right) \\
&\left(i d_{Z} \otimes c_{X, Y}\right)\left(y^{(-1)} \cdot z^{<0>(0)} \cdot S^{-1}\left(x^{<1>}\right) \otimes S^{-1}\left(z^{<0>(-1)}\right) \cdot x^{<0>} \otimes y^{(0)} \cdot z^{<1>}\right) \\
&= y^{(-1)} \cdot z^{<0>(0)} \cdot S^{-1}\left(x^{<1>}\right) \otimes\left[S^{-1}\left(z^{<0>(-1)}\right) \cdot x^{<0>}\right]^{(-1)} \cdot\left[y^{(0)} \cdot z^{<1>}\right]^{<0>} \\
& \otimes\left[S^{-1}\left(z^{<0>(-1)}\right) \cdot x^{<0>}\right]^{(0)} \cdot\left[y^{(0)} \cdot z^{<1>}\right]^{<1>} \\
& \underline{\underline{2.5 \mid 2.6}} y^{(-1)} \cdot z^{<0>(0)} \cdot S^{-1}\left(x^{<1>}\right) \otimes x^{<0>(-1)} \cdot y^{(0)<0>} \cdot z^{<1>} \\
& \otimes S^{-1}\left(z^{<0>(-1)}\right) \cdot x^{<0>(0)} \cdot y^{(0)<1>},
\end{aligned}
$$

and the two terms are equal because of the bicomodule condition for $X, Y$ and $Z$.

\section{Radford's Hopf algebras $H_{\nu}$}

Let $\nu$ be an odd natural number and assume that the base field $k$ contains a primitive $2 \nu^{\text {th }}$ root of unity $\omega$ and $2 \nu$ is invertible in $k$. We consider a certain family of Hopf algebras, which are exactly the quasitriangular ones from the larger family constructed by Radford in [12]. Namely, using notation as in [3], we denote by $H_{\nu}$ the Hopf algebra over $k$ generated by two elements $g$ and $x$ such that

$$
g^{2 \nu}=1, \quad g x+x g=0, \quad x^{2}=0,
$$

with coproduct $\Delta(g)=g \otimes g$ and $\Delta(x)=x \otimes g^{\nu}+1 \otimes x$, and antipode $S(g)=g^{-1}$ and $S(x)=g^{\nu} x$. Note that $H_{1}$ is exactly Sweedler's 4-dimensional Hopf algebra, and in general $H_{\nu}$ is $4 \nu$-dimensional, a linear basis in $H_{\nu}$ being the set $\left\{g^{l} x^{m} / 0 \leq l<2 \nu, 0 \leq m \leq 1\right\}$.

The quasitriangular structures of $H_{\nu}$ have been determined in [12]; they are parametrized by pairs $(s, \beta)$, where $\beta \in k$ and $s$ is an odd number with $1 \leq s<2 \nu$. Moreover, if we denote by $R_{s, \beta}$ the quasitriangular structure corresponding to $(s, \beta)$, then we have

$$
R_{s, \beta}=\frac{1}{2 \nu}\left(\sum_{i, l=0}^{2 \nu-1} \omega^{-i l} g^{i} \otimes g^{s l}\right)+\frac{\beta}{2 \nu}\left(\sum_{i, l=0}^{2 \nu-1} \omega^{-i l} g^{i} x \otimes g^{s l+\nu} x\right)
$$

It was also proved in [12] that $R_{s, \beta}$ is triangular if and only if $s=\nu$.

Following [12, we introduce an alternative description of $R_{s, \beta}$, more appropriate for our purpose. For every natural number $0 \leq l \leq 2 \nu-1$, we define

$$
e_{l}=\frac{1}{2 \nu} \sum_{i=0}^{2 \nu-1} \omega^{-i l} g^{i}
$$

regarded as an element in the group algebra of the cyclic group of order $2 \nu$ generated by the element $g$ (which in turn may be regarded as a Hopf subalgebra of $H_{\nu}$ in the obvious way). Then, by [12], the following relations hold:

$$
1=e_{0}+e_{1}+\ldots+e_{2 \nu-1}
$$




$$
\begin{aligned}
e_{i} e_{j} & =\delta_{i j} e_{i}, \\
g^{i} e_{j} & =\omega^{i j} e_{j},
\end{aligned}
$$

for all $0 \leq i, j \leq 2 \nu-1$. Also, a straightforward computation shows that we have

$$
\sum_{i=0}^{2 \nu-1}(-1)^{i} e_{i}=g^{\nu}
$$

Note also that, since $\omega$ is a primitive $2 \nu^{\text {th }}$ root of unity, we have

$$
\omega^{\nu}=-1
$$

With this notation, the quasitriangular structure $R_{s, \beta}$ may be expressed (cf. [12]) as

$$
R_{s, \beta}=\sum_{l=0}^{2 \nu-1} e_{l} \otimes g^{s l}+\beta\left(\sum_{l=0}^{2 \nu-1} e_{l} x \otimes g^{s l+\nu} x\right) .
$$

We are interested to see for what $s, \beta$ is $R_{s, \beta}$ pseudotriangular. We note first that for $\beta=0$, $R_{s, 0}$ is actually a quasitriangular structure on the group algebra of the cyclic group of order $2 \nu$, which is a commutative Hopf algebra, so $R_{s, 0}$ is pseudotriangular.

Consider now $R_{s, \beta}$ an arbitrary quasitriangular structure on $H_{\nu}$. We need to compute first $\left(R_{s, \beta}\right)_{21} R_{s, \beta}$. By using the defining relations $x^{2}=0$ and $g x+x g=0$, the properties of the elements $e_{l}$ listed above and the fact that $s$ and $\nu$ are odd numbers, a straightforward computation yields:

$$
\begin{aligned}
\left(R_{s, \beta}\right)_{21} R_{s, \beta}= & \sum_{l, t=0}^{2 \nu-1} \omega^{2 s l t} e_{l} \otimes e_{t}+\beta\left(\sum_{l, t=0}^{2 \nu-1} \omega^{2 s l t+\nu t} e_{l} x \otimes e_{t} x\right) \\
& -\beta\left(\sum_{l, t=0}^{2 \nu-1}(-1)^{l+t} \omega^{2 s l t+\nu l} x e_{l} \otimes e_{t} x\right) .
\end{aligned}
$$

Let us denote this element by $T$. We need to compare $T_{12} T_{23}$ and $T_{23} T_{12}$, so we first compute them, using repeatedly the defining relations of $H_{\nu}$ and the properties of the elements $e_{l}$ :

$$
\begin{aligned}
T_{12} T_{23}= & \sum_{l, t, j=0}^{2 \nu-1} \omega^{2 s l t+2 s t j} e_{l} \otimes e_{t} \otimes e_{j}+\beta\left(\sum_{l, t, i, j=0}^{2 \nu-1} \omega^{2 s l t+2 s i j+\nu j} e_{l} \otimes e_{t} e_{i} x \otimes e_{j} x\right. \\
& -\sum_{l, t, i, j=0}^{2 \nu-1}(-1)^{i+j} \omega^{2 s l t+2 s i j+\nu i} e_{l} \otimes e_{t} x e_{i} \otimes e_{j} x+\sum_{l, t, i, j=0}^{2 \nu-1} \omega^{2 s l t+2 s i j+\nu t} e_{l} x \otimes e_{t} x e_{i} \otimes e_{j} \\
& \left.-\sum_{l, t, i, j=0}^{2 \nu-1}(-1)^{t+l} \omega^{2 s l t+2 s i j+\nu l} x e_{l} \otimes e_{t} x e_{i} \otimes e_{j}\right) \\
= & \sum_{l, t, j=0}^{2 \nu-1} \omega^{2 s l t+2 s t j} e_{l} \otimes e_{t} \otimes e_{j}+\beta\left(\sum_{l, t, j=0}^{2 \nu-1}(-1)^{j} \omega^{2 s l t+2 s t j} e_{l} \otimes e_{t} x \otimes e_{j} x\right. \\
& -\sum_{l, t, i, j=0}^{2 \nu-1}(-1)^{j} \omega^{2 s l t+2 s i j} e_{l} \otimes e_{t} x e_{i} \otimes e_{j} x+\sum_{l, t, i, j=0}^{2 \nu-1}(-1)^{t} \omega^{2 s l t+2 s i j} e_{l} x \otimes e_{t} x e_{i} \otimes e_{j}
\end{aligned}
$$




$$
\begin{aligned}
& \left.-\sum_{l, t, i, j=0}^{2 \nu-1}(-1)^{t} \omega^{2 s l t+2 s i j} x e_{l} \otimes e_{t} x e_{i} \otimes e_{j}\right) \\
& =\sum_{l, t, j=0}^{2 \nu-1} \omega^{2 s l t+2 s t j} e_{l} \otimes e_{t} \otimes e_{j}+\beta\left(\sum_{l, t, j=0}^{2 \nu-1}(-1)^{j} \omega^{2 s l t} e_{l} \otimes e_{t} x \otimes g^{2 s t} e_{j} x\right. \\
& -\sum_{l, t, i, j=0}^{2 \nu-1}(-1)^{j} g^{2 s t} e_{l} \otimes e_{t} x e_{i} \otimes g^{2 s i} e_{j} x+\sum_{l, t, i, j=0}^{2 \nu-1}(-1)^{t} \omega^{2 s i j} e_{l} x \otimes g^{2 s l} e_{t} x e_{i} \otimes e_{j} \\
& \left.-\sum_{l, t, i, j=0}^{2 \nu-1}(-1)^{t} \omega^{2 s i j} x e_{l} \otimes g^{2 s l} e_{t} x e_{i} \otimes e_{j}\right) \\
& =\sum_{l, t, j=0}^{2 \nu-1} \omega^{2 s l t+2 s t j} e_{l} \otimes e_{t} \otimes e_{j}+\beta\left(\sum_{l, t=0}^{2 \nu-1} \omega^{2 s l t} e_{l} \otimes e_{t} x \otimes g^{2 s t+\nu} x\right. \\
& -\sum_{t, i=0}^{2 \nu-1} g^{2 s t} \otimes e_{t} x e_{i} \otimes g^{2 s i+\nu} x+\sum_{l, i, j=0}^{2 \nu-1} \omega^{2 s i j} e_{l} x \otimes g^{2 s l+\nu} x e_{i} \otimes e_{j} \\
& \left.-\sum_{l, i, j=0}^{2 \nu-1} \omega^{2 s i j} x e_{l} \otimes g^{2 s l+\nu} x e_{i} \otimes e_{j}\right) \\
& =\sum_{l, t, j=0}^{2 \nu-1} \omega^{2 s l t+2 s t j} e_{l} \otimes e_{t} \otimes e_{j}+\beta\left(\sum_{l, t=0}^{2 \nu-1} g^{2 s t} e_{l} \otimes e_{t} x \otimes g^{2 s t+\nu} x\right. \\
& -\sum_{t, i=0}^{2 \nu-1} g^{2 s t} \otimes e_{t} x e_{i} \otimes g^{2 s i+\nu} x+\sum_{l, i, j=0}^{2 \nu-1} e_{l} x \otimes g^{2 s l+2 s j+\nu} x e_{i} \otimes e_{j} \\
& \left.-\sum_{l, i, j=0}^{2 \nu-1} x e_{l} \otimes g^{2 s l+2 s j+\nu} x e_{i} \otimes e_{j}\right) \\
& =\sum_{l, t, j=0}^{2 \nu-1} \omega^{2 s l t+2 s t j} e_{l} \otimes e_{t} \otimes e_{j}+\beta\left(\sum_{t=0}^{2 \nu-1} g^{2 s t} \otimes e_{t} x \otimes g^{2 s t+\nu} x\right. \\
& -\sum_{t, i=0}^{2 \nu-1} g^{2 s t} \otimes e_{t} x e_{i} \otimes g^{2 s i+\nu} x+\sum_{l, j=0}^{2 \nu-1} e_{l} x \otimes g^{2 s l+2 s j+\nu} x \otimes e_{j} \\
& \left.-\sum_{l, j=0}^{2 \nu-1} x e_{l} \otimes g^{2 s l+2 s j+\nu} x \otimes e_{j}\right) \\
& T_{23} T_{12}=\sum_{l, t, j=0}^{2 \nu-1} \omega^{2 s l t+2 s t j} e_{l} \otimes e_{t} \otimes e_{j}+\beta\left(\sum_{l, t, j=0}^{2 \nu-1}(-1)^{t} \omega^{2 s l t+2 s t j} e_{l} x \otimes e_{t} x \otimes e_{j}\right. \\
& -\sum_{l, t, j=0}^{2 \nu-1}(-1)^{t} \omega^{2 s l t+2 s t j} x e_{l} \otimes e_{t} x \otimes e_{j}+\sum_{l, t, i, j=0}^{2 \nu-1}(-1)^{j} \omega^{2 s l t+2 s i j} e_{l} \otimes e_{i} x e_{t} \otimes e_{j} x \\
& \left.-\sum_{l, t, j=0}^{2 \nu-1}(-1)^{j} \omega^{2 s l t+2 s t j} e_{l} \otimes x e_{t} \otimes e_{j} x\right)
\end{aligned}
$$




$$
\begin{aligned}
= & \sum_{l, t, j=0}^{2 \nu-1} \omega^{2 s l t+2 s t j} e_{l} \otimes e_{t} \otimes e_{j}+\beta\left(\sum_{l, t, j=0}^{2 \nu-1}(-1)^{t} e_{l} x \otimes g^{2 s l+2 s j} e_{t} x \otimes e_{j}\right. \\
& -\sum_{l, t, j=0}^{2 \nu-1}(-1)^{t} x e_{l} \otimes g^{2 s l+2 s j} e_{t} x \otimes e_{j}+\sum_{l, t, i, j=0}^{2 \nu-1}(-1)^{j} \omega^{2 s l i+2 s t j} e_{l} \otimes e_{t} x e_{i} \otimes e_{j} x \\
& \left.-\sum_{l, t, j=0}^{2 \nu-1}(-1)^{j} \omega^{2 s l t} e_{l} \otimes x e_{t} \otimes g^{2 s t} e_{j} x\right) \\
= & \sum_{l, t, j=0}^{2 \nu-1} \omega^{2 s l t+2 s t j} e_{l} \otimes e_{t} \otimes e_{j}+\beta\left(\sum_{l, j=0}^{2 \nu-1} e_{l} x \otimes g^{2 s l+2 s j+\nu} x \otimes e_{j}\right. \\
& -\sum_{l, j=0}^{2 \nu-1} x e_{l} \otimes g^{2 s l+2 s j+\nu} x \otimes e_{j}+\sum_{l, t, i, j=0}^{2 \nu-1}(-1)^{j} g^{2 s i} e_{l} \otimes e_{t} x e_{i} \otimes g^{2 s t} e_{j} x \\
& \left.-\sum_{l, t=0}^{2 \nu-1} g^{2 s t} e_{l} \otimes x e_{t} \otimes g^{2 s t+\nu} x\right) \\
& \sum_{l, t, j=0}^{2 \nu-1} \omega^{2 s l t+2 s t j} e_{l} \otimes e_{t} \otimes e_{j}+\beta\left(\sum_{l, j=0}^{2 \nu-1} e_{l} x \otimes g^{2 s l+2 s j+\nu} x \otimes e_{j}\right. \\
& -\sum_{l, j=0}^{2 \nu-1} x e_{l} \otimes g^{2 s l+2 s j+\nu} x \otimes e_{j}+\sum_{t, i=0}^{2 \nu-1} g^{2 s i} \otimes e_{t} x e_{i} \otimes g^{2 s t+\nu} x \\
& \left.-\sum_{t=0}^{2 \nu-1} g^{2 s t} \otimes x e_{t} \otimes g^{2 s t+\nu} x\right) .
\end{aligned}
$$

Thus, we can see that we have

$$
\begin{aligned}
T_{12} T_{23}-T_{23} T_{12}= & \beta\left(\sum_{t=0}^{2 \nu-1} g^{2 s t} \otimes e_{t} x \otimes g^{2 s t+\nu} x-\sum_{t, i=0}^{2 \nu-1} g^{2 s t} \otimes e_{t} x e_{i} \otimes g^{2 s i+\nu} x\right. \\
& \left.-\sum_{t, i=0}^{2 \nu-1} g^{2 s i} \otimes e_{t} x e_{i} \otimes g^{2 s t+\nu} x+\sum_{t=0}^{2 \nu-1} g^{2 s t} \otimes x e_{t} \otimes g^{2 s t+\nu} x\right) .
\end{aligned}
$$

We need to prove now that we have

$$
x e_{l}=e_{l-\nu} x,
$$

for all $0 \leq l \leq 2 \nu-1$, where the subscripts are taken $\bmod 2 \nu$. We use the following facts:

$$
\begin{aligned}
& \omega^{\nu}=-1, \\
& x g^{i}=(-1)^{i} g^{i} x=\omega^{i \nu} g^{i} x .
\end{aligned}
$$

We have:

$$
x e_{l}=x \frac{1}{2 \nu} \sum_{i=0}^{2 \nu-1} \omega^{-i l} g^{i}
$$




$$
\begin{aligned}
& =\frac{1}{2 \nu} \sum_{i=0}^{2 \nu-1} \omega^{-i l} x g^{i} \\
& =\frac{1}{2 \nu} \sum_{i=0}^{2 \nu-1} \omega^{-i l} \omega^{i \nu} g^{i} x \\
& =\frac{1}{2 \nu} \sum_{i=0}^{2 \nu-1} \omega^{-i l+i \nu} g^{i} x \\
& =\frac{1}{2 \nu} \sum_{i=0}^{2 \nu-1} \omega^{-i(l-\nu)} g^{i} x \\
& =e_{l-\nu} x, \quad \text { q.e.d. }
\end{aligned}
$$

Now we compute:

$$
\begin{aligned}
\sum_{t, i=0}^{2 \nu-1} g^{2 s t} \otimes e_{t} x e_{i} \otimes g^{2 s i+\nu} x & =\sum_{t, i=0}^{2 \nu-1} g^{2 s t} \otimes e_{t} e_{i-\nu} x \otimes g^{2 s i+\nu} x \\
& =\sum_{t, i=0}^{2 \nu-1} g^{2 s t} \otimes \delta_{t, i-\nu} e_{t} x \otimes g^{2 s i+\nu} x \\
& =\sum_{t=0}^{2 \nu-1} g^{2 s t} \otimes e_{t} x \otimes g^{2 s(t+\nu)+\nu} x \\
& =\sum_{t=0}^{2 \nu-1} g^{2 s t} \otimes e_{t} x \otimes g^{2 s t+\nu} g^{2 s \nu} x \\
& =\sum_{t=0}^{2 \nu-1} g^{2 s t} \otimes e_{t} x \otimes g^{2 s t+\nu} x
\end{aligned}
$$

so we have $\sum_{t=0}^{2 \nu-1} g^{2 s t} \otimes e_{t} x \otimes g^{2 s t+\nu} x-\sum_{t, i=0}^{2 \nu-1} g^{2 s t} \otimes e_{t} x e_{i} \otimes g^{2 s i+\nu} x=0$. Similarly, we have:

$$
\begin{aligned}
\sum_{t, i=0}^{2 \nu-1} g^{2 s i} \otimes e_{t} x e_{i} \otimes g^{2 s t+\nu} x & =\sum_{t, i=0}^{2 \nu-1} g^{2 s i} \otimes x e_{t+\nu} e_{i} \otimes g^{2 s t+\nu} x \\
& =\sum_{t, i=0}^{2 \nu-1} g^{2 s i} \otimes x \delta_{t+\nu, i} e_{i} \otimes g^{2 s t+\nu} x \\
& =\sum_{i=0}^{2 \nu-1} g^{2 s i} \otimes x e_{i} \otimes g^{2 s(i-\nu)+\nu} x \\
& =\sum_{i=0}^{2 \nu-1} g^{2 s i} \otimes x e_{i} \otimes g^{2 s i+\nu} x
\end{aligned}
$$

so we have $\sum_{t=0}^{2 \nu-1} g^{2 s t} \otimes x e_{t} \otimes g^{2 s t+\nu} x-\sum_{t, i=0}^{2 \nu-1} g^{2 s i} \otimes e_{t} x e_{i} \otimes g^{2 s t+\nu} x=0$. Consequently, we have $T_{12} T_{23}-T_{23} T_{12}=0$, and so we obtained: 
Theorem 3.1 Any quasitriangular structure $R_{s, \beta}$ on Radford's Hopf algebra $H_{\nu}$ is pseudotriangular.

\section{Hopf algebras with positive bases}

In this section the base field is assumed to be $\mathbb{C}$, the field of complex numbers.

We recall from [6] that a basis of a Hopf algebra over $\mathbb{C}$ is called positive if all the structure constants (for the unit, counit, multiplication, comultiplication and antipode) with respect to this basis are nonnegative real numbers. Also, a quasitriangular structure $R$ on a Hopf algebra having a positive basis $B$ is called positive in [7] if the coefficients of $R$ in the basis $B \otimes B$ are nonnegative real numbers. The finite dimensional Hopf algebras having a positive basis and the positive quasitriangular structures on them have been classified in [6], 7] as follows.

Let $G$ be a group (we denote by $e$ its unit). A unique factorization $G=G_{+} G_{-}$of $G$ consists of two subgroups $G_{+}$and $G_{-}$of $G$ such that any $g \in G$ can be written uniquely as $g=g_{+} g_{-}$, with $g_{+} \in G_{+}$and $g_{-} \in G_{-}$. By considering the inverse map, we can also write uniquely $g=\bar{g}_{-} \bar{g}_{+}$, with $\bar{g}_{-} \in G_{-}$and $\bar{g}_{+} \in G_{+}$.

Let $u \in G_{+}, x \in G_{-}$; then we can write uniquely

$$
\begin{aligned}
& x u=\left({ }^{x} u\right)\left(x^{u}\right), \text { with }{ }^{x} u \in G_{+} \text {and } x^{u} \in G_{-}, \\
& u x=\left({ }^{u} x\right)\left(u^{x}\right), \text { with }{ }^{u} x \in G_{-} \text {and } u^{x} \in G_{+} .
\end{aligned}
$$

So, we have the following actions of $G_{+}$and $G_{-}$on each other (from left and right):

$$
\begin{array}{ll}
G_{-} \times G_{+} \rightarrow G_{+}, & (x, u) \mapsto{ }^{x} u, \\
G_{-} \times G_{+} \rightarrow G_{-}, & (x, u) \mapsto x^{u}, \\
G_{+} \times G_{-} \rightarrow G_{-}, & (u, x) \mapsto{ }^{u} x, \\
G_{+} \times G_{-} \rightarrow G_{+}, & (u, x) \mapsto u^{x} .
\end{array}
$$

The relations between these actions and the decompositions $g=g_{+} g_{-}=\bar{g}_{-} \bar{g}_{+}$are:

$\bar{g}_{-} \bar{g}_{+}=g_{+} ; \bar{g}_{-}^{\bar{g}_{+}}=g_{-} ; g_{+}^{g_{-}}=\bar{g}_{+} ;{ }^{g_{+}} g_{-}=\bar{g}_{-} ;\left({ }^{g_{+}} g_{-}\right)\left(g_{+}^{g_{-}}\right)=g_{+} g_{-} ;\left({ }^{g_{-}} g_{+}\right)\left(g_{-}^{g_{+}}\right)=g_{-} g_{+}$.

Given a unique factorization $G=G_{+} G_{-}$of a finite group $G$, one can construct a finite dimensional Hopf algebra $H\left(G ; G_{+}, G_{-}\right)$, which is the vector space spanned by the set $G$ (we denote by $\{g\}$ an element $g \in G$ when it is regarded as an element in $\left.H\left(G ; G_{+}, G_{-}\right)\right)$with the following Hopf algebra structure:

multiplication: $\{g\}\{h\}=\delta_{g_{+} g_{-}, h_{+}}\left\{g h_{-}\right\}$

unit: $\quad 1=\sum_{g_{+} \in G_{+}}\left\{g_{+}\right\}$

comultiplication: $\Delta(\{g\})=\sum_{h_{+} \in G_{+}}\left\{g_{+} h_{+}^{-1}\left(h_{+} g_{-}\right)\right\} \otimes\left\{h_{+} g_{-}\right\}$

counit: $\quad \varepsilon(\{g\})=\delta_{g_{+}, e}$

antipode: $\quad S(\{g\})=\left\{g^{-1}\right\}$

The Hopf algebra $H\left(G ; G_{+}, G_{-}\right)$has $G$ as the obvious positive basis. Conversely, it was proved in [6] that all finite dimensional Hopf algebras with positive bases are of the form $H\left(G ; G_{+}, G_{-}\right)$.

The positive quasitriangular and triangular structures on $H\left(G ; G_{+}, G_{-}\right)$have been described in [7] as follows:

Theorem 4.1 ([7]) Let $G=G_{+} G_{-}$be a unique factorization of a finite group $G$. Let $\xi, \eta$ : $G_{+} \rightarrow G_{-}$be two group homomorphisms satisfying the following conditions:

$$
\xi(u)^{v}=\xi\left(u^{\eta(v)}\right),
$$




$$
\begin{aligned}
& { }^{u} \eta(v)=\eta\left({ }^{\xi(u)} v\right), \\
& u v=\left({ }^{\xi(u)} v\right)\left(u^{\eta(v)}\right), \\
& \xi\left({ }^{x} u\right) x^{u}=x \xi(u), \\
& \eta\left({ }^{x} u\right) x^{u}=x \eta(u),
\end{aligned}
$$

for all $u, v \in G_{+}$and $x \in G_{-}$. Then

$$
R(\xi, \eta):=\sum_{u, v \in G_{+}}\left\{u\left(\eta(v)^{u}\right)^{-1}\right\} \otimes\{v \xi(u)\}
$$

is a positive quasitriangular structure on $H\left(G ; G_{+}, G_{-}\right)$. Conversely, every positive quasitriangular structure on $H\left(G ; G_{+}, G_{-}\right)$is given by the above construction.

Moreover, each of the conditions (4.1)-(4.5) is equivalent to the corresponding property below:

$$
\begin{aligned}
& { }^{v} \xi(u)=\xi\left({ }^{\eta(v)} u\right), \\
& \eta(v)^{u}=\eta\left(v^{\xi(u)}\right), \\
& u v=\left({ }^{\eta(u)} v\right)\left(u^{\xi(v)}\right), \\
& { }^{u} x \xi\left(u^{x}\right)=\xi(u) x, \\
& { }^{u} x \eta\left(u^{x}\right)=\eta(u) x .
\end{aligned}
$$

Moreover, $R(\xi, \eta)$ is triangular if and only if $\xi=\eta$.

Our aim now is to characterize those $R(\xi, \eta)$ that are pseudotriangular. So, let $R=R(\xi, \eta)$ be a positive quasitriangular structure on $H\left(G ; G_{+}, G_{-}\right)$. We have (see [7]):

$$
R_{21} R=\sum_{u, v \in G_{+}}\left\{v \xi(u)\left(\eta(\bar{v})^{\bar{u}}\right)^{-1}\right\} \otimes\left\{u\left(\eta(v)^{u}\right)^{-1} \xi(\bar{u})\right\}
$$

where we denoted $\bar{u}=v^{\xi(u)}$ and $\bar{v}=\eta(v) u$.

We denote $T=R_{21} R$ and we compute (by using the formula for the multiplication of $\left.H\left(G ; G_{+}, G_{-}\right)\right)$:

$$
\begin{aligned}
T_{12} T_{23}= & \left(\sum_{u, v \in G_{+}}\left\{v \xi(u)\left(\eta(\bar{v})^{\bar{u}}\right)^{-1}\right\} \otimes\left\{u\left(\eta(v)^{u}\right)^{-1} \xi(\bar{u})\right\} \otimes 1\right) \\
& \left(\sum_{s, t \in G_{+}} 1 \otimes\left\{t \xi(s)\left(\eta(\bar{t})^{\bar{s}}\right)^{-1}\right\} \otimes\left\{s\left(\eta(t)^{s}\right)^{-1} \xi(\bar{s})\right\}\right) \\
= & \sum_{u, v, s, t \in G_{+}}\left\{v \xi(u)\left(\eta(\bar{v})^{\bar{u}}\right)^{-1}\right\} \otimes\left\{u\left(\eta(v)^{u}\right)^{-1} \xi(\bar{u})\right\}\left\{t \xi(s)\left(\eta(\bar{t})^{\bar{s}}\right)^{-1}\right\} \\
& \left.\otimes\left\{s\left(\eta(t)^{s}\right)^{-1} \xi(\bar{s})\right\}\right) \\
= & \sum_{u, v, s \in G_{+}}\left\{v \xi(u)\left(\eta(\eta(v) u)^{\left(v^{\xi(u)}\right)}\right)^{-1}\right\} \otimes\left\{u\left(\eta(v)^{u}\right)^{-1} \xi\left(v^{\xi(u)}\right) \xi(s)\left(\eta\left({ }^{(t)} s\right)^{\left(t^{\xi(s)}\right)}\right)^{-1}\right\} \\
& \otimes\left\{s\left(\eta(t)^{s}\right)^{-1} \xi\left(t^{\xi(s)}\right)\right\},
\end{aligned}
$$

where $t=u^{\left(\eta(v)^{u}\right)^{-1} \xi\left(v^{\xi(u)}\right)}$, and

$$
T_{23} T_{12}=\left(\sum_{a, b \in G_{+}} 1 \otimes\left\{b \xi(a)\left(\eta\left({ }^{(b)} a\right)^{\left(b^{\xi(a)}\right)}\right)^{-1}\right\} \otimes\left\{a\left(\eta(b)^{a}\right)^{-1} \xi\left(b^{\xi(a)}\right)\right\}\right)
$$




$$
\begin{aligned}
& \left(\sum_{c, d \in G_{+}}\left\{d \xi(c)\left(\eta\left({ }^{\eta(d)} c\right)^{\left(d^{\xi(c)}\right)}\right)^{-1}\right\} \otimes\left\{c\left(\eta(d)^{c}\right)^{-1} \xi\left(d^{\xi(c)}\right)\right\} \otimes 1\right) \\
= & \sum_{a, b, c, d \in G_{+}}\left\{d \xi(c)\left(\eta\left(^{\eta(d)} c\right)^{\left(d^{\xi(c)}\right)}\right)^{-1}\right\} \otimes\left\{b \xi(a)\left(\eta\left({ }^{(b)} a\right)^{\left(b^{\xi(a)}\right)}\right)^{-1}\right\}\left\{c\left(\eta(d)^{c}\right)^{-1} \xi\left(d^{\xi(c)}\right)\right\} \\
= & \sum_{a, b, d \in G_{+}}\left\{d \xi(c)\left(\eta\left({ }^{a}\right)^{-1} \xi\left(b^{\xi(a)}\right)\right\}\right. \\
& \otimes\left\{a\left(\eta(b)^{a}\right)^{-1} \xi\left(b^{\xi(a)}\right)\right\},
\end{aligned}
$$

where $c=b^{\xi(a)\left(\eta\left({ }^{\eta(b)} a\right)^{\left(b^{\xi(a)}\right)}\right)^{-1}}$. By writing down what means $T_{12} T_{23}=T_{23} T_{12}$, we obtain:

Proposition 4.2 The positive quasitriangular structure $R(\xi, \eta)$ is pseudotriangular if and only if the following conditions are satisfied:

$$
\begin{aligned}
& \xi(u)\left(\eta\left(^{\eta(v)} u\right)^{\left(v^{\xi(u)}\right)}\right)^{-1}=\xi(c)\left(\eta\left({ }^{(v)} c\right)^{\left(v^{\xi(c)}\right)}\right)^{-1}, \\
& \left(\eta(v)^{u}\right)^{-1} \xi\left(v^{\xi(u)}\right) \xi(s)\left(\eta\left(^{\eta(t)} s\right)^{\left(t^{\xi(s)}\right)}\right)^{-1}=\xi(s)\left(\eta(\eta(u) s)^{\left(u^{\xi(s)}\right)}\right)^{-1}\left(\eta(v)^{c}\right)^{-1} \xi\left(v^{\xi(c)}\right), \\
& \left(\eta(t)^{s}\right)^{-1} \xi\left(t^{\xi(s)}\right)=\left(\eta(u)^{s}\right)^{-1} \xi\left(u^{\xi(s)}\right),
\end{aligned}
$$

for all $u, v, s \in G_{+}$, where $t=u^{\left(\eta(v)^{u}\right)^{-1} \xi\left(v^{\xi(u)}\right)}$ and $\left.\left.c=u^{\xi(s)(\eta(\eta(u)} s\right)^{\left(u^{\xi(s)}\right)}\right)^{-1}$.

A better description may be obtained for a certain class of positive quasitriangular structures.

Definition 4.3 ([7]) A positive quasitriangular structure $R(\xi, \eta)$ on $H\left(G ; G_{+}, G_{-}\right)$is called normal if $\xi(u)=e$ for all $u \in G_{+}$.

Theorem 4.4 A normal positive quasitriangular structure $R(\xi, \eta)$ on $H\left(G ; G_{+}, G_{-}\right)$is pseudotriangular if and only if $\eta(u v)=\eta(v u)$ for all $u, v \in G_{+}$.

Proof. We note first that, since $\xi(u)=e$ for all $u \in G_{+}$, some of the relations (4.1)-(4.10) may be simplified, in particular we have ${ }^{u} \eta(v)=\eta(v), u v=v\left(u^{\eta(v)}\right), \eta(v)^{u}=\eta(v), u v=\left({ }^{\eta(u)} v\right) u$, for all $u, v \in G_{+}$. By using these relations, together with the fact that $\xi(u)=e$ for all $u \in G_{+}$, the three conditions in the above Proposition may be also simplified, so we obtain that $R(\xi, \eta)$ is pseudotriangular if and only if we have:

$$
\begin{aligned}
& \eta\left(v u v^{-1}\right)=\eta\left(v c v^{-1}\right), \\
& \eta(v)^{-1} \eta\left(t s t^{-1}\right)^{-1}=\eta\left(u s u^{-1}\right)^{-1} \eta(v)^{-1} \\
& \eta(t)^{-1}=\eta(u)^{-1}
\end{aligned}
$$

for all $u, v, s \in G_{+}$, where $t=v u v^{-1}$ and $c=u s u s^{-1} u^{-1}$, and one can easily see that each of these three conditions is equivalent to the condition $\eta(u v)=\eta(v u)$, for all $u, v \in G_{+}$.

We recall from [9] that the canonical quasitriangular structure on the Drinfeld double of a finite dimensional Hopf algebra $H$ is pseudotriangular if and only if $H$ is commutative and cocommutative. In particular, if $G$ is a finite group, the canonical quasitriangular structure on the Drinfeld double of the dual $k[G]^{*}$ of the group algebra $k[G]$ is pseudotriangular if and only if $G$ is abelian. We want to reobtain this result (over $\mathbb{C}$ ) as an application of Theorem 4.4, 
We consider the unique factorization $G=G_{+} G_{-}$, where $G_{+}=G$ and $G_{-}=\{e\}$ (so the Hopf algebra $H\left(G ; G_{+}, G_{-}\right)$is exactly $\left.k[G]^{*}\right)$. As in [7, we consider the group $\tilde{G}=G \times G$, with the unique factorization $\tilde{G}=\tilde{G}_{+} \tilde{G}_{-}$, where $\tilde{G}_{+}=G \times\{e\}$ and $\tilde{G}_{-}=\{(g, g): g \in G\}$. By [7], the group homomorphisms $\xi, \eta: \tilde{G}_{+} \rightarrow \tilde{G}_{-}$defined by $\xi(g, e)=(e, e)$ and $\eta(g, e)=(g, g)$ induce a positive quasitriangular structure $R(\xi, \eta)$ on $H\left(\tilde{G} ; \tilde{G}_{+}, \tilde{G}_{-}\right)$and moreover $H\left(\tilde{G} ; \tilde{G}_{+}, \tilde{G}_{-}\right)$is the Drinfeld double of $H\left(G ; G_{+}, G_{-}\right)=k[G]^{*}$ and $R(\xi, \eta)$ is its canonical quasitriangular structure. Obviously $R(\xi, \eta)$ is normal, so we may apply Theorem 4.4 and we obtain that $R(\xi, \eta)$ is pseudotriangular if and only if $(g h, g h)=(h g, h g)$ for all $g, h \in G$, i.e. if and only if $G$ is abelian.

\section{$5 \quad$ Universality of the pseudosymmetric category $\mathcal{P} \mathcal{S}$}

In this section we use terminology, notation and some results from [5] (but we use the term "monoidal" instead of "tensor" when we speak about tensor categories and tensor functors).

Our aim is to show that the pseudosymmetric category $\mathcal{P S}$ introduced in [10] has two universality properties similar to the ones of the braid category $\mathcal{B}$, the universal braided monoidal category (see [5]). First, we recall from [10] the definition of $\mathcal{P} \mathcal{S}$. The objects of $\mathcal{P} \mathcal{S}$ are natural numbers $n \in \mathbb{N}$. The set of morphisms from $m$ to $n$ is empty if $m \neq n$ and is $P S_{n}:=\frac{B_{n}}{\left[P_{n}, P_{n}\right]}$ if $m=n$, where $B_{n}$ (respectively $P_{n}$ ) is the braid group (respectively pure braid group) on $n$ strands. The monoidal structure of $\mathcal{P} \mathcal{S}$ is defined as the one for $\mathcal{B}$, and so is the braiding, namely (we denote as usual by $\sigma_{1}, \sigma_{2}, \ldots, \sigma_{n-1}$ the standard generators of $B_{n}$ and by $\pi_{n}$ the natural morphism from $B_{n}$ to $\left.P S_{n}\right)$ :

$$
\begin{gathered}
c_{n, m}: n \otimes m \rightarrow m \otimes n, \quad c_{0, n}=i d_{n}=c_{n, 0}, \\
c_{n, m}=\pi_{n+m}\left(\left(\sigma_{m} \sigma_{m-1} \cdots \sigma_{1}\right)\left(\sigma_{m+1} \sigma_{m} \cdots \sigma_{2}\right) \cdots\left(\sigma_{m+n-1} \sigma_{m+n-2} \cdots \sigma_{n}\right)\right) \text { if } m, n>0 .
\end{gathered}
$$

In order to introduce the first universality property for $\mathcal{P} \mathcal{S}$, we need the following definition, motivated by results in [10] and by the definition of Yang-Baxter operators from [5]:

Definition 5.1 If $V$ is an object in a monoidal category $(\mathcal{C}, \otimes, I, a, l, r)$, an automorphism $\sigma$ of $V \otimes V$ is called a pseudosymmetric Yang-Baxter operator on $V$ if the following two dodecagons 
(for $\sigma$ and $\left.\sigma^{-1}\right)$ commute:

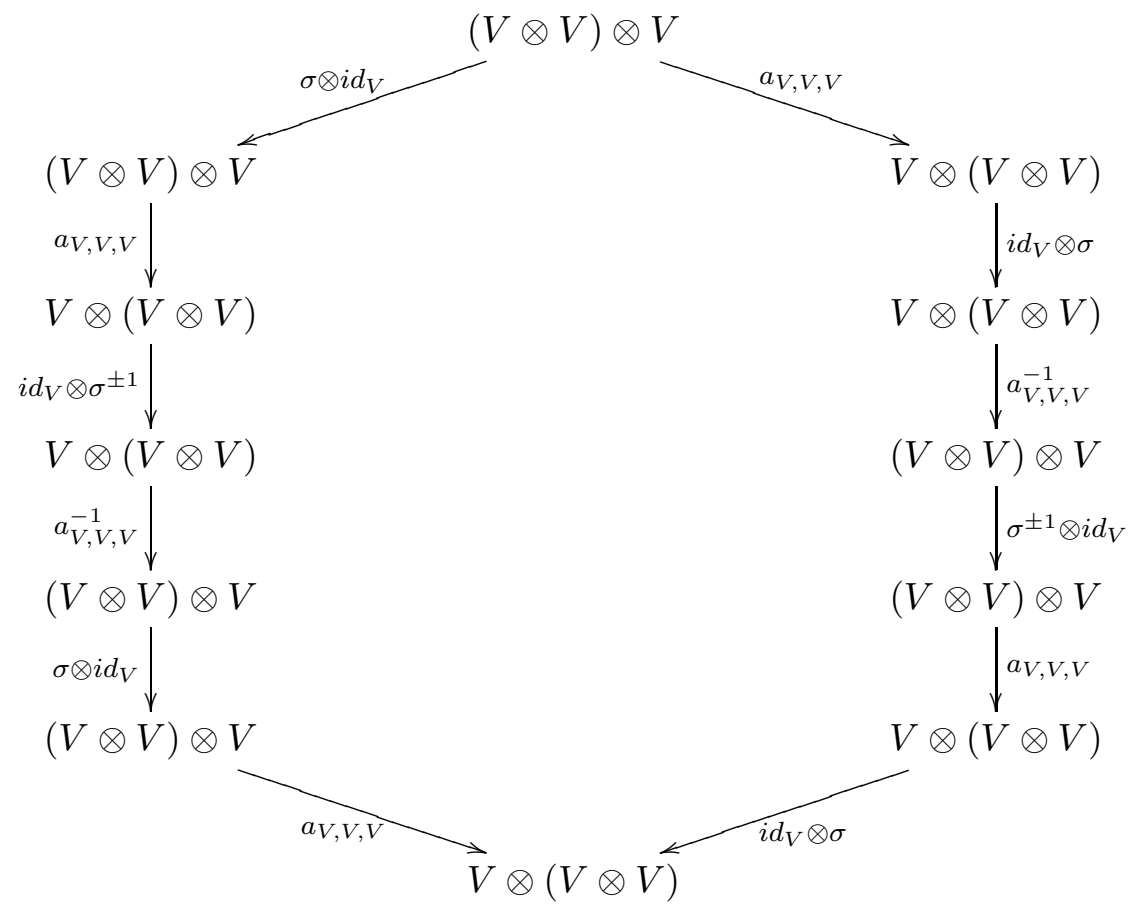

Note that a pseudosymmetric Yang-Baxter operator is a special type of Yang-Baxter operator as defined in [5], p. 323. Moreover, just like Yang-Baxter operators, they can be transferred by using functors between monoidal categories:

Lemma 5.2 Let $\left(F, \varphi_{0}, \varphi_{2}\right): \mathcal{C} \rightarrow \mathcal{D}$ be a monoidal functor between two monoidal categories. If $\sigma \in \operatorname{Aut}(V \otimes V)$ is a pseudosymmetric Yang-Baxter operator on the object $V \in \mathcal{C}$, then

$$
\sigma^{\prime}=\varphi_{2}(V, V)^{-1} \circ F(\sigma) \circ \varphi_{2}(V, V)
$$

is a pseudosymmetric Yang-Baxter operator on $F(V)$.

Proof. The proof follows exactly as in [5], Lemma XIII.3.2, by using also the identity

$$
\left(\sigma^{\prime}\right)^{-1}=\varphi_{2}(V, V)^{-1} \circ F\left(\sigma^{-1}\right) \circ \varphi_{2}(V, V)
$$

in order to prove the pseudosymmetry of $\sigma^{\prime}$.

We define the category $P S Y B(\mathcal{C})$ of pseudosymmetric Yang-Baxter operators to be a full subcategory of $Y B(\mathcal{C})$, the category of Yang-Baxter operators defined in [5]. An object in $P S Y B(\mathcal{C})$ is a pair $(V, \sigma)$ where $V$ is a object in $\mathcal{C}$ and $\sigma$ is a pseudosymmetric Yang-Baxter operator.

Recall the following construction from [5]. Suppose that $\left(F, \varphi_{0}, \varphi_{2}\right): \mathcal{B} \rightarrow \mathcal{C}$ is a monoidal functor from the universal braid category $\mathcal{B}$ to a given monoidal category $\mathcal{C}$. Since $c_{1,1}=\sigma_{1}$ is a Yang-Baxter operator on the object $1 \in \mathcal{B}$, it follows that $\sigma=\varphi_{2}^{-1}(1,1) F\left(c_{1,1}\right) \varphi_{2}(1,1)$ is a Yang-Baxter operator on $F(1) \in \mathcal{C}$. In this way we get a functor $\Theta: \operatorname{Tens}(\mathcal{B}, \mathcal{C}) \rightarrow Y B(\mathcal{C})$, where $\operatorname{Tens}(\mathcal{B}, \mathcal{C})$ is the category of monoidal functors from $\mathcal{B}$ to $\mathcal{C}$. It was proved in [5] that:

Theorem 5.3 ([5]) For any monoidal category $\mathcal{C}$, the functor $\Theta:$ Tens $(\mathcal{B}, \mathcal{C}) \rightarrow Y B(\mathcal{C})$ is an equivalence of categories. 
One can note that we have a natural monoidal functor $\pi: \mathcal{B} \rightarrow \mathcal{P S}$ induced by the group epimorphism $\pi_{n}: B_{n} \rightarrow P S_{n}$. This allows us to identify the category $\operatorname{Tens}(\mathcal{P S}, \mathcal{C})$ with a subcategory of $\operatorname{Tens}(\mathcal{B}, \mathcal{C})$. More precisely, we identify it with the full subcategory of all monoidal functors $F: \mathcal{B} \rightarrow \mathcal{C}$ with the property that there exists a monoidal functor $G: \mathcal{P S} \rightarrow \mathcal{C}$ such that $F=G \circ \pi$.

We can state now the first universality property of $\mathcal{P} \mathcal{S}$ :

Theorem 5.4 For any monoidal category $\mathcal{C}$, the functor $\widetilde{\Theta}: \operatorname{Tens}(\mathcal{P} \mathcal{S}, \mathcal{C}) \rightarrow \operatorname{PSYB}(\mathcal{C}), \widetilde{\Theta}(G)=$ $\Theta(G \circ \pi)$ is an equivalence of categories.

Proof. First we note that $\pi\left(c_{1,1}\right)$ is a pseudosymmetric Yang-Baxter operator in $\mathcal{P} \mathcal{S}$ and so by Lemma 5.2 we have $\varphi_{2}^{-1}(1,1) G\left(\pi\left(c_{1,1}\right)\right) \varphi_{2}(1,1) \in P S Y B(\mathcal{C})$. This means that $\widetilde{\Theta}$ is well defined. Since $\Theta$ is fully faithful and $\widetilde{\Theta}$ is its restriction to a full subcategory, it is enough to show that $\widetilde{\Theta}$ is essentially surjective. This follows from the next lemma.

Lemma 5.5 Let $\mathcal{C}$ be a strict monoidal category and $(V, \sigma)$ an object in $P S Y B(\mathcal{C})$. Then there exists a unique strict monoidal functor $G: \mathcal{P S} \rightarrow \mathcal{C}$ such that $G(1)=V$ and $G\left(\pi\left(c_{1,1}\right)\right)=\sigma$.

Proof. From [5], Lemma XIII.3.5 we know that for all $(V, \sigma) \in Y B(\mathcal{C})$ there exists a unique strict monoidal functor $F: \mathcal{B} \rightarrow \mathcal{C}$ such that $F(1)=V$ and $F\left(c_{1,1}\right)=\sigma$. It is enough to show that when $(V, \sigma) \in P S Y B(\mathcal{C})$ the functor $F$ factors through $\pi$. But this follows immediately from the fact (see [10]) that

$$
P S_{n}=\frac{B_{n}}{<\sigma_{i} \sigma_{i+1}^{-1} \sigma_{i}=\sigma_{i+1} \sigma_{i}^{-1} \sigma_{i+1} \mid 1 \leq i \leq n-2>}
$$

and the definition of a pseudosymmetric Yang-Baxter operator.

Definition 5.6 ([5]) A monoidal functor $\left(F, \varphi_{0}, \varphi_{2}\right)$ from a braided monoidal category $\mathcal{C}$ to a braided monoidal category $\mathcal{D}$ is braided if for every pair $(U, V)$ of objects in $\mathcal{C}$ the square

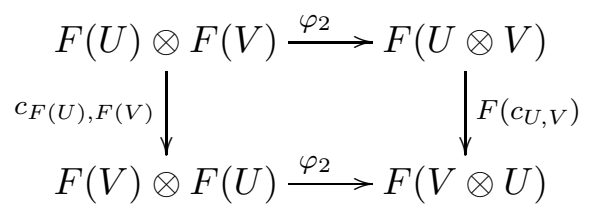

commutes. Denote by $\operatorname{Br}(\mathcal{C}, \mathcal{D})$ the category whose objects are braided monoidal functors and morphisms are natural monoidal transformations.

Theorem 5.7 ([5]) For a braided monoidal category $\mathcal{C}$, the functor $\Theta^{\prime}: \operatorname{Br}(\mathcal{B}, \mathcal{C}) \rightarrow \mathcal{C}$ defined by $\Theta^{\prime}(F)=F(1)$ is an equivalence of categories.

In the definition of a pseudosymmetric braided category $\mathcal{C}$ introduced in $[9$ was assumed that $\mathcal{C}$ was a strict monoidal category. The next proposition is the analogue of Theorem 3.7 from [9] for monoidal categories with nontrivial associativity constraints. Note that the proof that we present here is very direct and is inspired by the results in [10]. 
Proposition 5.8 Let $(\mathcal{C}, \otimes, I, a, l, r, c)$ be a braided monoidal category. The following conditions are equivalent:

(i) For every $U, V, W \in \mathcal{C}$ the following diagram is commutative:

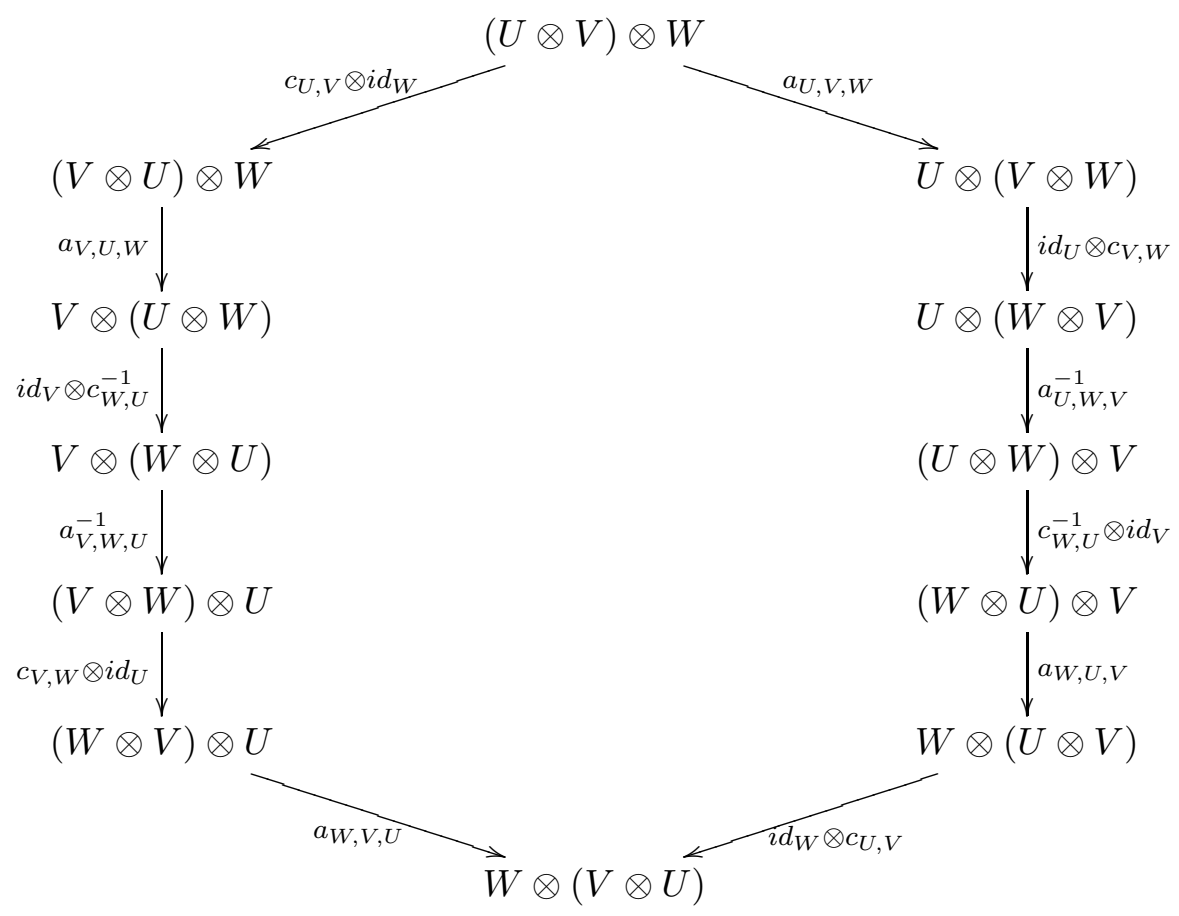

(ii) For every $U, V, W \in \mathcal{C}$ the following diagram is commutative:

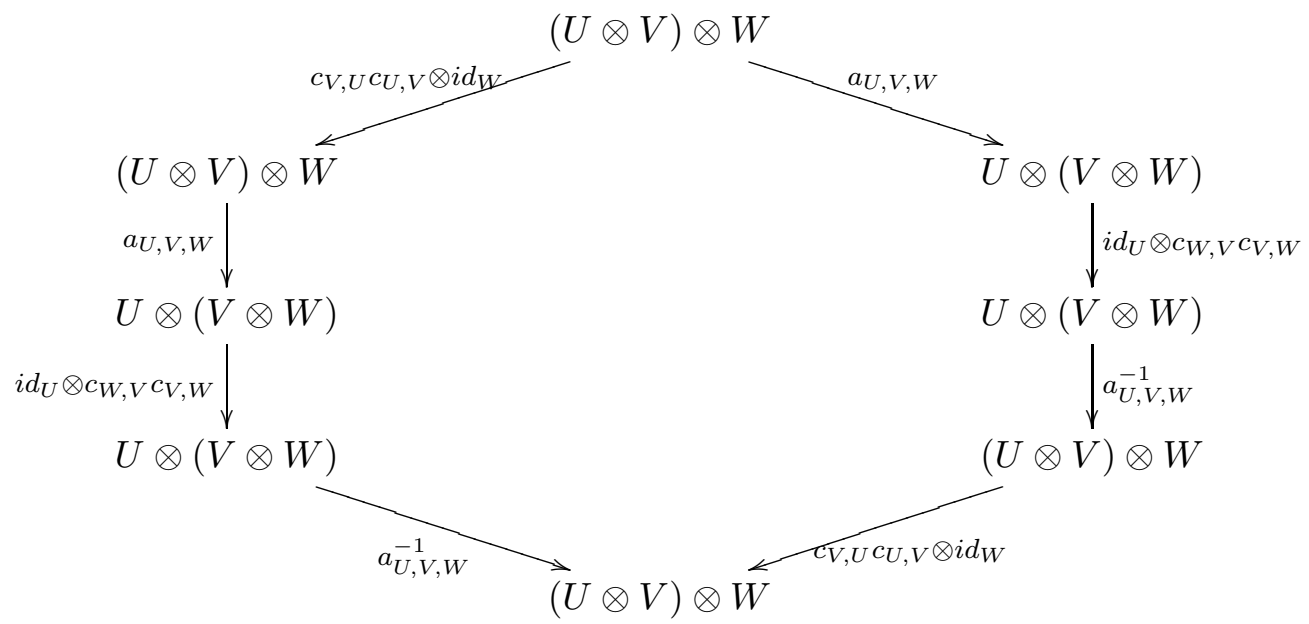

Proof. Take $U, V, W \in \mathcal{C}$. Using only the fact that $\mathcal{C}$ is a braided category we have

$$
\begin{aligned}
\left(\left(c_{V, U} c_{U, V}\right) \otimes i d_{W}\right) a_{U, V, W}^{-1}\left(i d_{U} \otimes\left(c_{W, V} c_{V, W}\right)\right) a_{U, V, W} \\
=\quad\left(c_{V, U} \otimes i d_{W}\right) a_{V, U, W}^{-1}\left(i d_{V} \otimes c_{U, W}^{-1}\right)\left[\left(i d_{V} \otimes c_{U, W}\right) a_{V, U, W}\left(c_{U, V} \otimes i d_{W}\right)\right] \\
\quad a_{U, V, W}^{-1}\left(i d_{U} \otimes c_{W, V} c_{V, W}\right) a_{U, V, W} \\
=\quad\left(c_{V, U} \otimes i d_{W}\right) a_{V, U, W}^{-1}\left(i d_{V} \otimes c_{U, W}^{-1}\right)\left[a_{V, W, U} c_{U, V \otimes W} a_{U, V, W}\right] a_{U, V, W}^{-1}
\end{aligned}
$$




$$
\begin{aligned}
&\left(i d_{U} \otimes c_{W, V} \circ c_{V, W}\right) a_{U, V, W} \\
&=\left(c_{V, U} \otimes i d_{W}\right) a_{V, U, W}^{-1}\left(i d_{V} \otimes c_{U, W}^{-1}\right) a_{V, W, U}\left(c_{W, V} \otimes i d_{U}\right)\left(c_{V, W} \otimes i d_{U}\right) c_{U, V \otimes W} a_{U, V, W}, \\
& a_{U, V, W}^{-1}\left(i d_{U} \otimes c_{W, V}\right)\left(i d_{U} \otimes c_{V, W}\right) a_{U, V, W}\left(c_{V, U} \otimes i d_{W}\right)\left(c_{U, V} \otimes i d_{W}\right) \\
&= a_{U, V, W}^{-1}\left(i d_{U} \otimes c_{W, V}\right) a_{U, W, V} c_{V, U \otimes W} a_{V, U, W}\left(c_{U, V} \otimes i d_{W}\right) \\
&= a_{U, V, W}^{-1}\left(i d_{U} \otimes c_{W, V}\right) a_{U, W, V} c_{V, U \otimes W}\left(i d_{V} \otimes c_{U, W}^{-1}\right)\left(i d_{V} \otimes c_{U, W}\right) a_{V, U, W}\left(c_{U, V} \otimes i d_{W}\right) \\
&= a_{U, V, W}^{-1}\left(i d_{U} \otimes c_{W, V}\right) a_{U, W, V}\left(c_{U, W}^{-1} \otimes i d_{V}\right) c_{V, W} \otimes U \\
&= a_{U, V, W}^{-1}\left(i d_{V} \otimes d_{U, W}\right) a_{V, U, W}\left(c_{U, V} \otimes i d_{W}\right) \\
&\left(i d_{V} \otimes c_{U, W}\right) a_{V, U, W}\left(c_{U, V} \otimes i d_{W}\right) \\
&= a_{U, V, W}^{-1}\left(i d_{U} \otimes c_{W, V}\right) a_{U, W, V}\left(c_{U, W}^{-1} \otimes i d_{V}\right) a_{W, U, V}^{-1}\left(i d_{W} \otimes c_{V, U}\right) a_{W, V, U}\left(c_{V, W} \otimes i d_{U}\right) a_{V, W, U}^{-1} \\
& a_{V, W, U} c_{U, V} \otimes W a_{U, V, W} \\
&= a_{U, V, W}^{-1}\left(i d_{U} \otimes c_{W, V}\right) a_{U, W, V}\left(c_{U, W}^{-1} \otimes i d_{V}\right) a_{W, U, V}^{-1}\left(i d_{W} \otimes c_{V, U}\right) a_{W, V, U} \\
&\left(c_{V, W} \otimes i d_{U}\right) c_{U, V \otimes W} a_{U, V, W} .
\end{aligned}
$$

This means that the condition (ii) holds if and only if

$$
\begin{aligned}
& \left(c_{V, U} \otimes i d_{W}\right) a_{V, U, W}^{-1}\left(i d_{V} \otimes c_{U, W}^{-1}\right) a_{V, W, U}\left(c_{W, V} \otimes i d_{U}\right) \\
& \quad=a_{U, V, W}^{-1}\left(i d_{U} \otimes c_{W, V}\right) a_{U, W, V}\left(c_{U, W}^{-1} \otimes i d_{V}\right) a_{W, U, V}^{-1}\left(i d_{W} \otimes c_{V, U}\right) a_{W, V, U}
\end{aligned}
$$

and this condition is obviously equivalent with (i).

Definition 5.9 We say that a braided monoidal category $(\mathcal{C}, \otimes, I, a, l, r, c)$ is pseudosymmetric if it satisfies any of the two equivalent conditions from Proposition 5.8 .

Remark 5.10 If $(\mathcal{C}, \otimes, I, a, l, r, c)$ is a pseudosymmetric braided monoidal category and $V$ is an object in $\mathcal{C}$, then $c_{V, V}$ is a pseudosymmetric Yang-Baxter operator on $V$.

Lemma 5.11 If the braided category $\mathcal{C}$ is pseudosymmetric then $\operatorname{Br}(\mathcal{B}, \mathcal{C}) \cong \operatorname{Br}(\mathcal{P S}, \mathcal{C})$.

Proof. The isomorphism is induced by $\pi: \mathcal{B} \rightarrow \mathcal{P S}$. More precisely, we have

$$
\pi^{*}: \operatorname{Br}(\mathcal{P S}, \mathcal{C}) \rightarrow \operatorname{Br}(\mathcal{B}, \mathcal{C}), \quad \pi^{*}(G)=G \circ \pi
$$

Because $\pi_{n}: B_{n} \rightarrow P S_{n}$ is surjective and the category $\mathcal{C}$ is pseudosymmetric, any functor $F \in \operatorname{Br}(\mathcal{B}, \mathcal{C})$ is of the form $F=G \circ \pi$ for some unique $G \in \operatorname{Br}(\mathcal{P S}, \mathcal{C})$.

As a consequence of this and Theorem 5.7 we obtain the second universality property of $\mathcal{P} \mathcal{S}$ :

Theorem 5.12 For a pseudosymmetric braided category $\mathcal{C}$, the functor $\widetilde{\Theta^{\prime}}: \operatorname{Br}(\mathcal{P} \mathcal{S}, \mathcal{C}) \rightarrow \mathcal{C}$ defined by $\widetilde{\Theta^{\prime}}(G)=G(1)$ is an equivalence of categories. 


\section{References}

[1] A. Bruguières, Double braidings, twists and tangle invariants, J. Pure Appl. Algebra 204 (2006), 170-194.

[2] D. Bulacu, S. Caenepeel, F. Panaite, Yetter-Drinfeld categories for quasi-Hopf algebras, Comm. Algebra 34 (2006), 1-35.

[3] G. Carnovale, J. Cuadra, The Brauer group of some quasitriangular Hopf algebras, J. Algebra 259 (2003), 512-532.

[4] A. Joyal, R. Street, Braided tensor categories, Adv. Math. 102 (1993), 20-78.

[5] C. Kassel, Quantum groups, Grad. Texts Math. 155, Springer-Verlag, Berlin, 1995.

[6] J.-H. Lu, M. Yan, Y.-C. Zhu, On Hopf algebras with positive bases, J. Algebra 237 (2001), 421-445.

[7] J.-H. Lu, M. Yan, Y.-C. Zhu, On quasitriangular structures on Hopf algebras with positive bases, in "New trends in Hopf algebra theory" (1999), Amer. Math. Soc., Providence, RI, pp. 339-356.

[8] F. Panaite, M. D. Staic, F. Van Oystaeyen, On some classes of lazy cocycles and categorical structures, J. Pure Appl. Algebra 209 (2007), 687-701.

[9] F. Panaite, M. D. Staic, F. Van Oystaeyen, Pseudosymmetric braidings, twines and twisted algebras, J. Pure Appl. Algebra 214 (2010), 867-884.

[10] F. Panaite, M. D. Staic, A quotient of the braid group related to pseudosymmetric braided categories, Pacific J. Math. 244 (2010), 155-167.

[11] F. Panaite, F. Van Oystaeyen, L-R-smash biproducts, double biproducts and a braided category of Yetter-Drinfeld-Long bimodules, Rocky Mount. J. Math. 40 (2010), 2013-2024.

[12] D. E. Radford, On Kauffmann's knot invariants arising from finite dimensional Hopf algebras, in "Advances in Hopf algebras", Lecture Notes Pure Appl. Math. Vol. 158, Dekker, New York, 1994, pp. 205-266.

[13] M. D. Staic, Pure-braided Hopf algebras and knot invariants, J. Knot Theory Ramifications 13 (2004), 385-400. 